\title{
Vorwort zur 8. Auflage
}

Die Stadt Nürnberg und die Markt- und Meinungsforschung sind eng miteinander verbunden. Professor Wilhelm Vershofen gründete hier im Jahr 1925 das Institut für Wirtschaftsbeobachtungen der deutschen Fertigware (IfW) und baute das erste organisatorische System zur Erfassung von Konsumbedürfnissen der deutschen Bevölkerung auf. Zahlreiche Marktforschungsinstitute mit quantitativer und qualitativer Ausrichtung wurden in Nürnberg gegründet. Heute sind zwanzig Prozent aller deutschen Marktforscher in Nürnberg tätig, wodurch die Bedeutung der Stadt als Marktforschungshochburg hervorgehoben wird.

Eine entsprechend große Tradition hat auch die Ausbildung von Marktforschern an den Hochschulen der Stadt. Das Berufsbild der Marktforscher erlebt dabei in den letzten Jahren einen radikalen Wandel. Die quantitative Marktforschung wurde weitgehend digitalisiert und ist um ein vielfaches schneller, effizienter und vielschichtiger geworden als in der Vergangenheit. Reine Zahlen liefern allerdings nach wie vor keine Insights und daher kommt der analytischen Kompetenz und dem Datenverständnis von Marktforschern eine zunehmende Bedeutung zu. Fachabteilungen in Unternehmen fordern von Marktforschern immer mehr projektbezogene Consumer Insights, um kurzfristig Entscheidungen treffen zu können. Daher haben sich auch in der Marktforschung agile Arbeitsmethoden durchgesetzt, bei denen ein Denken in Methodenbaukästen an die Stelle von starren Verfahrensabläufen tritt.

Entsprechend wurde auch das vorliegende Buch zur Förderung von Kompetenzen zukünftiger Markforscher in der 8. Auflage einem vollständigen Relaunch unterzogen. Der erste Teil zu den methodischen Grundlagen wurde komplett überarbeitet. Inhaltlich wurden neue Ansätze (Mobile App-Befragungen, Mixed-Mode-Designs, ...) und Branchen-Standards (DSGVO, ESOMAR, ... ) integriert. Didaktisch wurden die Inhalte über verbundene Youtube-Erklärvideos und Kontroll- bzw. Vertiefungsfragen erweitert. Die Anwendungsbeispiele in den Abschnitten zwei bis vier wurden nach Themenclustern entsprechend der üblichen Marketingplanung neu geordnet und auf den aktuellen Stand der Forschung gebracht. Für den abschließenden methodisch-analytischen Teil wurde ein SPSS-Datensatz entwickelt, anhand dessen alle Berechnungen transparent nachvollzogen werden können. An der Virtuellen Hochschule Bayern kann dieser Teil bei Interesse über das eigene Kursangebot „Marktforschung mit SPSS“ weiter vertieft werden. Die Youtube-Videos, der SPSS-Datensatz und viele weitere digitale Ergänzungsmaterialien können über QR-Codes und die begleitende Webseite zum Buch http://www.degruyter.com/view/title/568793 kostenfrei abgerufen werden.

Neu in der 8. Auflage war auch die konzentrierte Zusammenarbeit des Initiators dieses Standardwerks, Prof. Dr. Jörg Koch, mit dem aktuellen Marktforschungs-Professor der TH Nürnberg, Prof. Dr. Florian Riedmüller. Unser verdienter Kollege Prof. Dr. Peter Gebhardt ist mit seinem Eintritt in den Ruhestand aus dem Autorenteam aus- 
geschieden. Unverändert ist die hervorragende Zusammenarbeit mit dem De Gruyter Verlag und unserem Editor Dr. Stefan Giesen. Ein großer Dank geht an unsere Mitarbeiterin Frau Juliana Haas, die den Erstellungsprozess tatkräftig unterstützte.

Prof. Dres Jörg Koch und Florian Riedmüller

(joerg.koch@th-nuernberg.de; florian.riedmueller@th-nuernberg.de) 